\title{
Nanosecond Laser Treatment of a-Si Thin Films for Enhanced Light Trapping and Minority Carrier Lifetime in Photovoltaic Cells
}

\author{
Esther Blesso Vidhya Y and Nilesh J Vasa \\ Department of Engineering Design, Indian Institute of Technology, \\ Chennai,600036,India \\ E-mail: estherblessoanand@gmail.com
}

\begin{abstract}
For thin film solar cells, the efficient collection of light is a challenge, therefore an efficient light-trapping scheme, such as the textures, is required for achieving high light conversion efficiencies. Laser texturing with a Q-switched, nanosecond pulsed $\mathrm{Nd}^{3+}$ :YAG laser is used to create nano scale surface roughness in a-Si thin films that enhances light-trapping with simultaneous crystallization. Changes in surface morphology due to laser assisted texturing in air and water environments on 1- $\mu \mathrm{m}$ thick n-type amorphous silicon film deposited on p-type c-Si is observed using Optical profilometer and Atomic Force Microscopy. The light-trapping properties of textures are analyzed by optical reflectance measurements and compared with theoretical analysis. The extent of crystallinity and the influence of structural characteristics on electronic properties are studied using I-V characterization and open circuit voltage decay method. Reduction in reflection is observed compared to planar surface indicating dependence of reflectance on surface textures which is in agreement with the theoretical analysis. After underwater annealing, efficiency of n-aSi/p-cSi solar cells is found to be increased ( $2 \%$ to $3 \%$ ) when compared to that in air and diffusion lengths of minority carriers above $1-\mu \mathrm{m}$ after laser treatment indicates the reduction in the probability of recombination before crossing the junction.
\end{abstract}

DOI: 10.2961/jlmn.2017.03.0009

Keywords: laser annealing, amorphous silicon, texturing, thin films, solar cells, light trapping, minority carrier life time, diffusion length, finite element method

\section{Introduction}

Crystalline silicon is the most commonly used material for industrial bulk solar-cells due to its efficiency, stability and availability. Thin film solar cells are gaining importance because of the reduction in the amount of active material and formation of tandem junctions [1]. Amorphous silicon is a low cost material which is used for electronic devices like thin film transistors [TFTs] and photo voltaic devices. It has high absorption coefficient and the energy gap can be modified to improve the light conversion efficiency; tandem junctions can be formed with other elements like carbon and germanium. It can be deposited on a variety of materials at low temperature, over large areas, and on flexible substrates like plastics and polymers [2]. However in amorphous silicon, the absence of crystalline structure and the random network of atoms causes anomalous electronic behaviour resulting in low photovoltaic efficiency. Efficiency can be improved by crystallizing the aSi film by thermal annealing method, such as laser annealing technique [3].

Light trapping is important for thin film solar cells where a single light-pass through the absorber is not sufficient to capture the weakly absorbed red and near-infrared photons. The amount of light absorbed depends on the optical path length and the absorption coefficient. Textured surface reduces the reflections and increases the optical path length and enhances the optical absorption by lighttrapping. This can be achieved by simultaneous formation of laser induced surface texture while annealing [4].
Conventionally, excimer lasers, such as $\mathrm{XeCl}(308 \mathrm{~nm})$, $\mathrm{KrF}(248 \mathrm{~nm})$ lasers have been used for annealing of thin film solar cell $[5,6]$. Due to strong absorption at the surface, they can only be used for the annealing of thin film of few tens of nanometers. Laser-induced crystallization and texturing of the film of few hundreds of nm to $\approx 1000 \mathrm{~nm}$ needed for solar applications is a difficult task with excimer lasers. In addition, they have certain limitations, such as high operational cost and environmental problems due to its toxic nature. Alternatively, $\mathrm{Nd}^{3+}$ :YAG laser with the fundamental wavelength $(1064 \mathrm{~nm})$, the second $(532 \mathrm{~nm})$ and the third (355 $\mathrm{nm}$ ) harmonics can be considered for the treatment of a-Si films [7].

Laser surface texturing of crystalline silicon has been reported $[8,9]$, however, there are limited work on simultaneous annealing and texturing of a-Si thin films in air and water[10]. Most of the studies reported use femtosecond lasers, which involve ablation induced surface structuring. Simultaneous wide-area annealing and texturing of thick (> few hundred $\mathrm{nm}$ ) a-Si films in air and water ambience using laser-assisted melting and re-solidification approach without ablation is a challenging task. This approach induces the texture formation via capillary action in molten silicon without ablating the material [4].

In our earlier works, we reported the effect of laser fluence, beam profile, laser wavelength and ambience on the surface morphology of the thin film and the extent of crystallinity with various characterization techniques [11,12] In this paper, the formation of light trapping structures and 

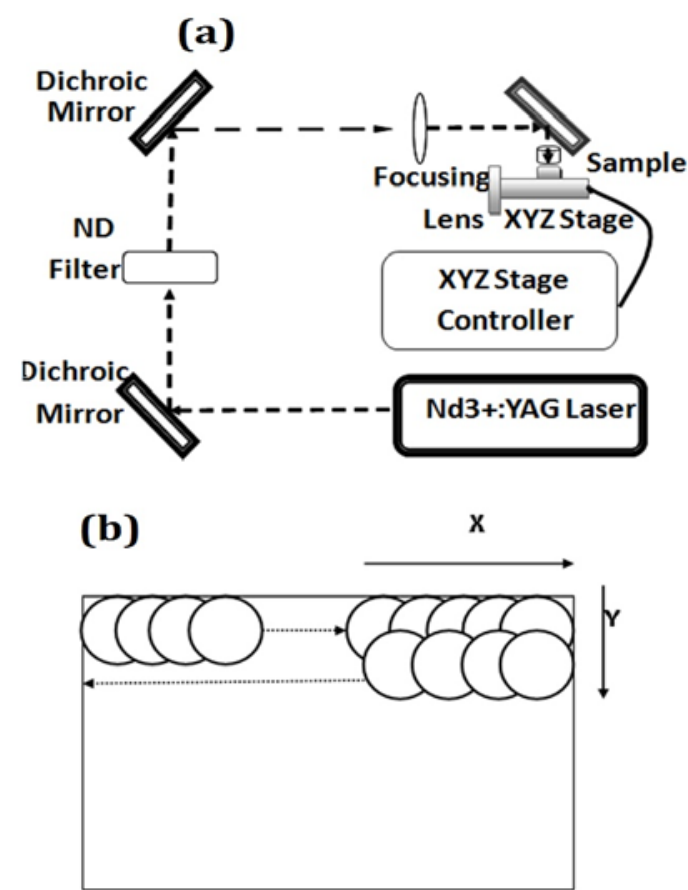

Fig. 1 (a) Experimental setup for nanosecond Laser annealing (b) Laser spot overlap.

crystallization characteristics of a-Si thin film annealed and textured using the solid-state $\mathrm{Nd}^{3+}$ :YAG laser in air and water environment is studied. The dependence of properties like reflectance, transmittance and absorptance on surface textures are analyzed by numerical simulations based on Maxwell's wave equations with periodic surface textures with different dimensions and density. The extent of crystallinity and the influence of structural characteristics on electronic properties are studied using I-V characterization and the calculation of minority carrier life time and diffusion length using the open circuit voltage decay method.

\section{Experiment}

Experiments are performed in different ambience such as in air and water to determine appropriate conditions for annealing and texturing of a-Si films. P-type (Boron doped) crystalline silicon wafers of $275 \pm 25 \mu \mathrm{m}$ thickness coated with n-type (Phosphorous doped) amorphous silicon films of $0.4 \mu \mathrm{m}$ and $1 \mu \mathrm{m}$ thickness by plasmaenhanced chemical vapor deposition technique (Hind High Vacuum Co., India) using silane (SiH4), Hydrogen and 1\% phosphine in silane at 1 torr pressure and $40 \mathrm{~mW} / \mathrm{cm}^{2}$ power density are used in these experiments.

Figure 1 shows the schematic of the experimental setup. Laser annealing is performed using a solid-state, pulsed $\mathrm{Nd}^{3+}$ :YAG laser (Brilliant b, Quantel) with a wavelength of $532 \mathrm{~nm}$ and $355 \mathrm{~nm}$, pulse duration of $5 \mathrm{~ns}$ (FWHM) and pulse repetition rate of $1 \mathrm{~Hz}$. A plano-convex Lens with a focal length of $200 \mathrm{~mm}$ is used to form a $2 \mathrm{~mm}$ laser spot size on a-Si film. Distance between the lens and sample was adjusted to obtain the same laser spot size in air and water. The laser beam with a Gaussian and a flat-top beam profile were used in the experiments. The Gaussian beam profile was changed into the flat-top beam profile using a dispersion based beam shaper ( $\pi$ Shaper 12_12_532_HP, AdlOptica). To determine the fluence range for laser melting, surface reflectivity measurements are performed with a red diode laser (635 $\mathrm{nm}$ ) as a probe laser as reported in our earlier work[4]. For under water experiments samples are kept at $3 \mathrm{~mm}$ depth in the deionized water. $10 \mathrm{~mm} \times 10$ mm samples are scanned with laser spot overlapping for simultaneous crystallization and texturization of wide area a-Si, with $30 \%, 50 \%$ and $90 \%$ of the spot diameter as an overlap as shown in figure 1(b).

The formation light trapping textures are studied by a scanning electron microscope (SEM), optical profilometry (Bruker, 3D Non-contact Profiler, Contour GT-I) and the (a)

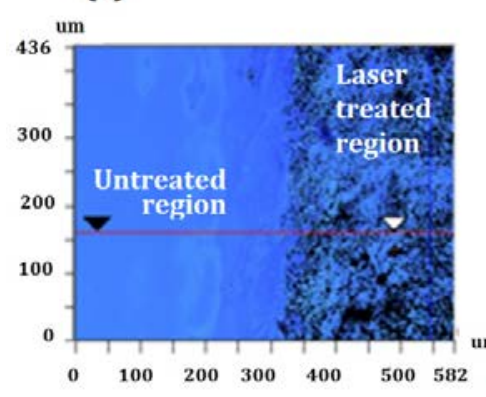

(b)

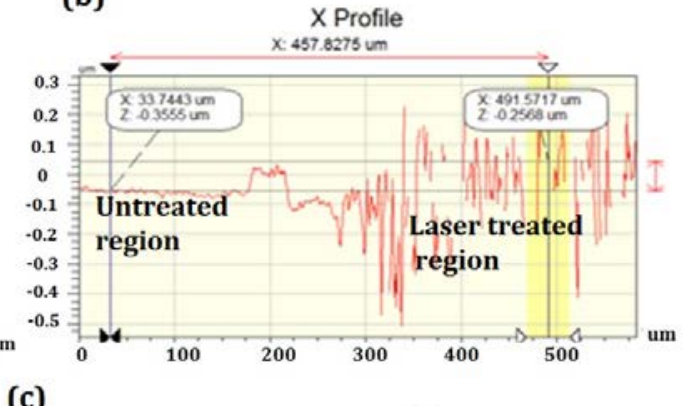

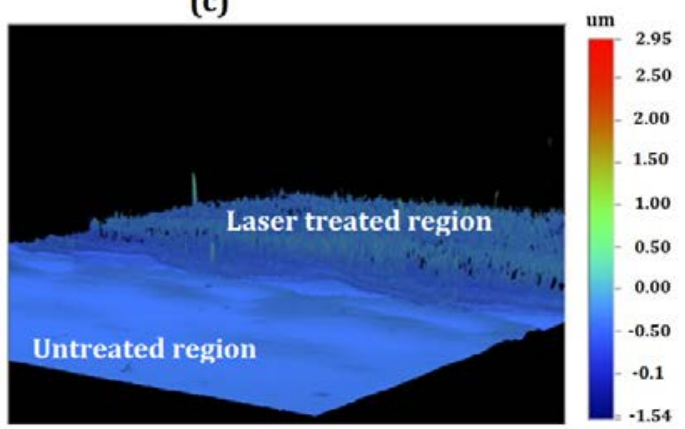

Fig. 2 Surface Profile of a-Si/c-Si sample showing regions of untreated and treated with ns-laser at $532 \mathrm{~nm}$ with 150 $\mathrm{mJ} / \mathrm{cm}^{2}$ (a),(b) 2D image and its roughness profile (c) 3D profile (The left region in each image is untreated region). 
atomic force microscopy (AFM) (Bruker, Dimension edge) to analyze the texturing effect. The effect of light trapping textures on optical reflectance is analyzed using a broadband light source and a monochromator system (Bentham PVE 300). The effect of crystallization on the electronic properties are studied using $\mathrm{I}-\mathrm{V}$ characterization and the open circuit voltage decay method using a white light Xe lamp and an AM 1.5 filter with a digital storage oscilloscope (Lecroy, wave runner, $250 \mathrm{MHz}$ ) and electrical source/ measurement unit (B2901A, Keysight).

\section{Theoretical analysis}

In this numerical analysis, geometrical structures are theoretically investigated on a nanometer scale to improve the optical properties of the textured surface of Si solar cells. Maxwell's equations, are solved to model the optical properties of surfaces that have been textured. This employs full wave finite element method to simulate the interaction of the incident light with nanostructure, which is realized by infinitely extending the unit texture using periodic boundary conditions. Then the electromagnetic field distribution can be obtained through resolving Maxwell's equations. Thereby, the spatial distribution of the energy flux, and thus the optical characteristics such as reflection, transmission, and absorption spectra, can be obtained by integrating the calculated electromagnetic field [13].

The simulations are carried out using the RF module of COMSOL 5.2 Multiphysics software package. The optical properties of materials are described by complex wavelength-dependent refraction index. The simulation is carried out for TE polarized wave and at wavelengths in the range of absorption of Si (300 $\mathrm{nm}$ to $1100 \mathrm{~nm}$ ). 2-D periodic textures are considered in the simulations. The dimensions of the texture period and height have been varied based on the experimental observations.
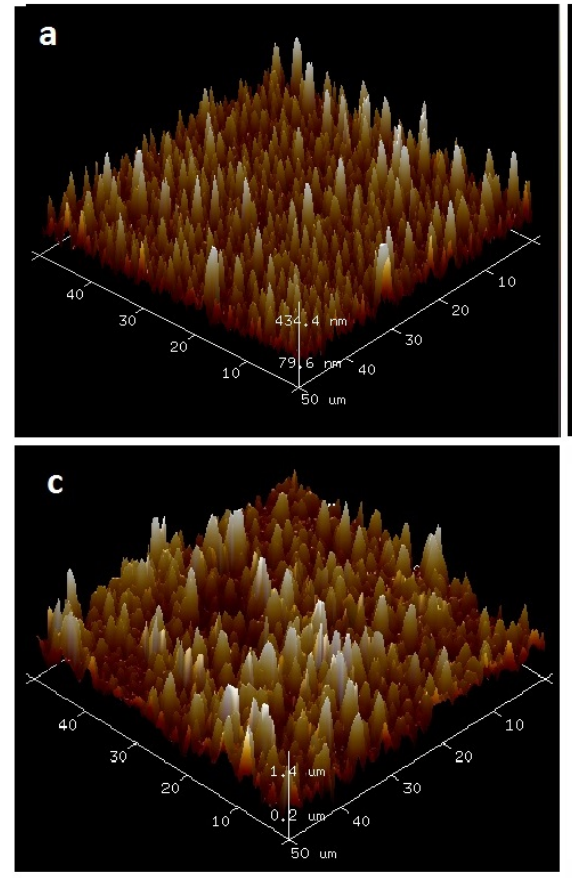

\section{Results and discussions}

\subsection{Light trapping}

Laser-assisted melting and resolidification approach induces the texture formation via capillary action in molten silicon without ablating the material. As the laser interacts with the material surface, the temperature rises with possible phase changes. Due to the surface tension, capillary waves are generated. The capillary waves will exist in the liquefied region until silicon starts cooling due to thermal conductivity and the waves are frozen to change the surface topology. These structures subsequently sharpen into spikes by repeated overlapping of pulses.

Figure 2 shows the surface characteristics of laser treated a-Si films, by using the optical profilometer. Fig. 2(a) and (c) shows the 2D and 3D surface roughness profile of the sample and (b) shows the surface profile along red line in Fig. 2(a) (X-direction). Left region of in Figs. 2(a) and (b) show the untreated region where the surface is smooth and the treated region on the right clearly indicates the formation of textures after laser treatment. Based on the optical profilometer studies, in the case of the Gaussian beam annealing at the laser fluence of $150 \mathrm{~mJ} / \mathrm{cm}^{2}$ in air, an average height of $300 \mathrm{~nm}$ texture formation is observed.

Figures 3(a), (b) and (c) show AFM images of the laser textured a-Si film samples with $30 \%, 50 \%$ and $90 \%$ of beam overlap corresponding to the Gaussian beam profile in air with $355 \mathrm{~nm}$ wavelength, respectively. The density and uniformity of texture peaks are increased with the flattop beam profile and water ambience [4]. Root mean square (RMS) value of surface roughness (Rq parameter) is estimated from AFM images. Figure 3(d) shows variation of surface roughness (Rq) values of the samples shown in Figs. 3(a) to (c) compared with the samples treated with flat-top beam profiles. The increase in surface roughness is
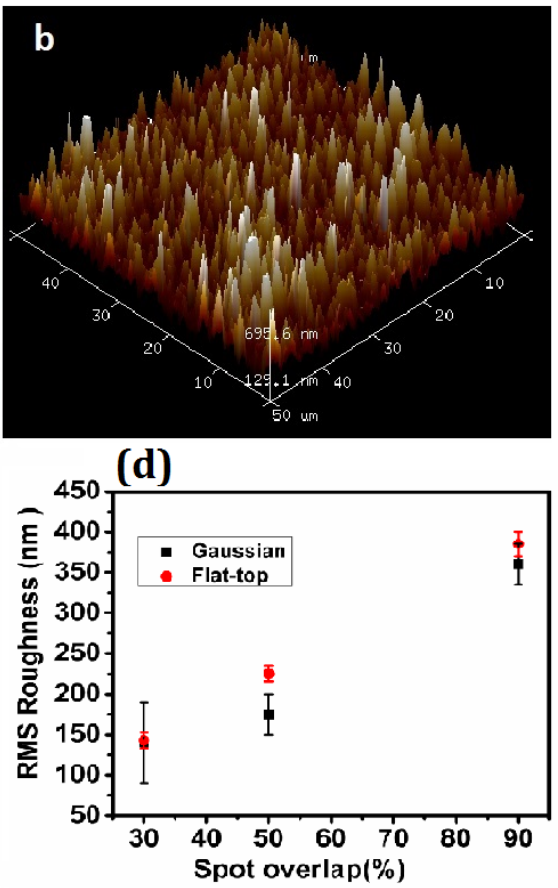

Fig. 3 AFM images of laser treated a-Si/c-Si with Gaussian beam profile in air and (a) $30 \%$ (b) 50\% and (c) 90\% of laser spot overap and (d) RMS roughness with Gaussian and flat-top beams with a fluence of $300 \mathrm{~mJ} / \mathrm{cm}^{2}$. 


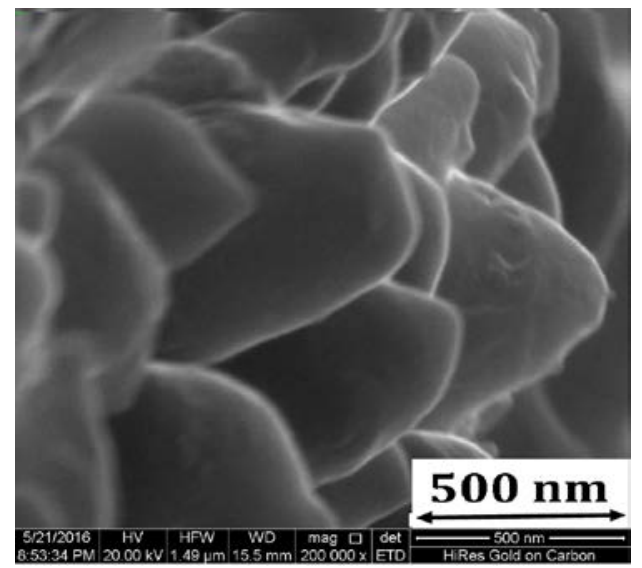

Fig. 4 SEM micrograph of cross sectional view of a-Si/c-Si sample treated with $532 \mathrm{~nm}$ with fluence value of 300 $\mathrm{mJ} / \mathrm{cm}^{2}$ with $90 \%$ spot overlapping with flat-top beam profile in water.

observed for the increase in laser spot overlap. From the comparative investigation with 50\% and 90\% overlap for a$\mathrm{Si} / \mathrm{c}-\mathrm{Si}$ samples, it is observed that, the samples with $90 \%$ overlap and treated in air show higher $\mathrm{Rq}$ values. In the case of flat-top beam profile with a lower percentage of overlap, the textures are almost uniform and the surface roughness variation is negligible throughout the film surface whereas with Gaussian beam profile significant surface roughness variation is observed. As percentage of overlap increased, the uniformity of textures with Gaussian beam profiles also increased.

To view the micro structural change in the a-Si thin film after laser crystallization, cross sectional view of thin film is observed using a scanning electron microscope (FEG Quanta 400) with a magnification of 20000×. Significant difference in surface could be seen between the treated and untreated samples. Conical structures are observed after laser texturing as shown in Figure 4. Underwater annealing

(a)

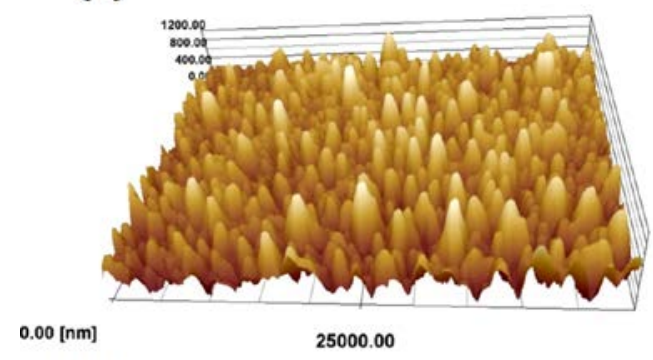

(b)

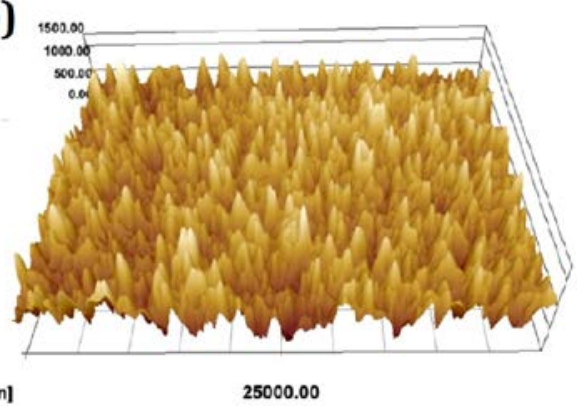

Fig. 5 AFM images of laser treated a-Si/c-Si with Gaussian beam profile in (a) air and (b) water with the laser fluence of $300 \mathrm{~mJ} / \mathrm{cm}^{2}$ and laser spot overlap of $30 \%$ with $532 \mathrm{~nm}$. produces small base high density structures compared to

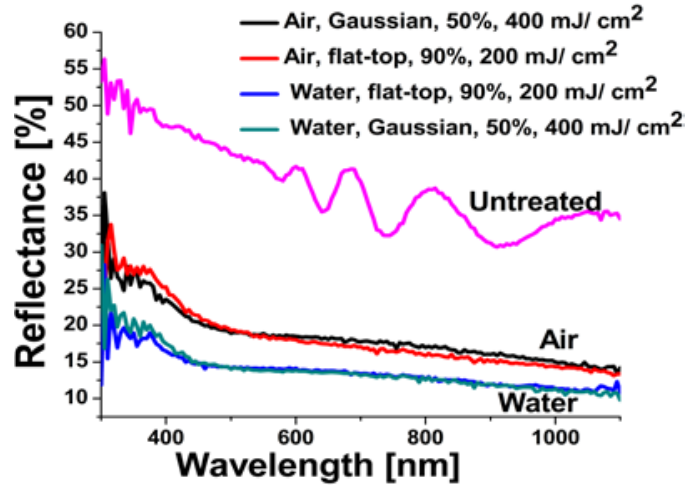

Fig. 6 Optical reflectance of the untreated and laser treated a$\mathrm{Si} / \mathrm{c}$-Si samples under different conditions.

annealing in air environment as shown in Fig. 5. This smaller size formation in water must be due to a reduction in capillary wavelength in water ambience [14,15].The molten silicon layer solidify quicker in water than in air, since thermal conductivity and heat capacity of liquid water were greater than those of air. The dispersion relation for capillary waves in a shallow layer of molten silicon indicates that reduction in the lifetime of the liquid layer de-

Table: 1 Laser induced texture dimensions under various treated condition

\begin{tabular}{|c|c|c|c|c|}
\hline Ambience & $\begin{array}{c}\text { Laser } \\
\text { spot } \\
\text { overlap } \\
\text { (\%) }\end{array}$ & $\begin{array}{c}\text { Beam } \\
\text { profile }\end{array}$ & $\begin{array}{c}{ }^{*} \text { Cone } \\
\text { height }(\mathrm{nm})\end{array}$ & $\begin{array}{l}\text { *Cone base } \\
\text { diameter } \\
(\mathrm{nm})\end{array}$ \\
\hline \multirow{6}{*}{ Air } & 30 & Gaussian & $150-310$ & $1500-2500$ \\
\hline & & Flat-top & $100-300$ & $1500-2500$ \\
\hline & 50 & Gaussian & $150-350$ & $1500-2000$ \\
\hline & & Flat-top & $160-300$ & $1500-2250$ \\
\hline & 90 & Gaussian & $170-690$ & $1500-2500$ \\
\hline & & Flat-top & $170-320$ & $1200-2000$ \\
\hline \multirow{6}{*}{ Water } & 30 & Gaussian & $130-290$ & $1000-1600$ \\
\hline & & Flat-top & $120-200$ & $1000-1500$ \\
\hline & 50 & Gaussian & $150-370$ & $1000-1500$ \\
\hline & & Flat-top & $140-300$ & $1000-1800$ \\
\hline & 90 & Gaussian & $200-600$ & $1000-2000$ \\
\hline & & Flat-top & $150-350$ & $900-1600$ \\
\hline
\end{tabular}

* Data for laser fluence varying from $200 \mathrm{~mJ} / \mathrm{cm}^{2}$ to $400 \mathrm{~mJ} / \mathrm{cm}^{2}$

creases the longest allowed capillary wavelength. Since the cooling time was reduced by the flow of heat to the surrounding water in underwater laser treatment, it results in smaller and high dense structures.

After confirming the formation of textures due to laser annealing, the light trapping effect due to these textures on solar cell has been investigated by optical reflectance measurements. Figure 6 shows optical reflectance spectra of the thin-film surface in the wavelength region of 300 to $1100 \mathrm{~nm}$ measured using a broadband light source and a monochromator with an integrating sphere. The reflectance values across the whole spectra reduced by 20 to $35 \%$ compared to untreated sample indicating an improvement in light trapping via multiple reflections due to the textured surface. Compared to air treated samples, water treated samples show high reduction in reflection which might be 

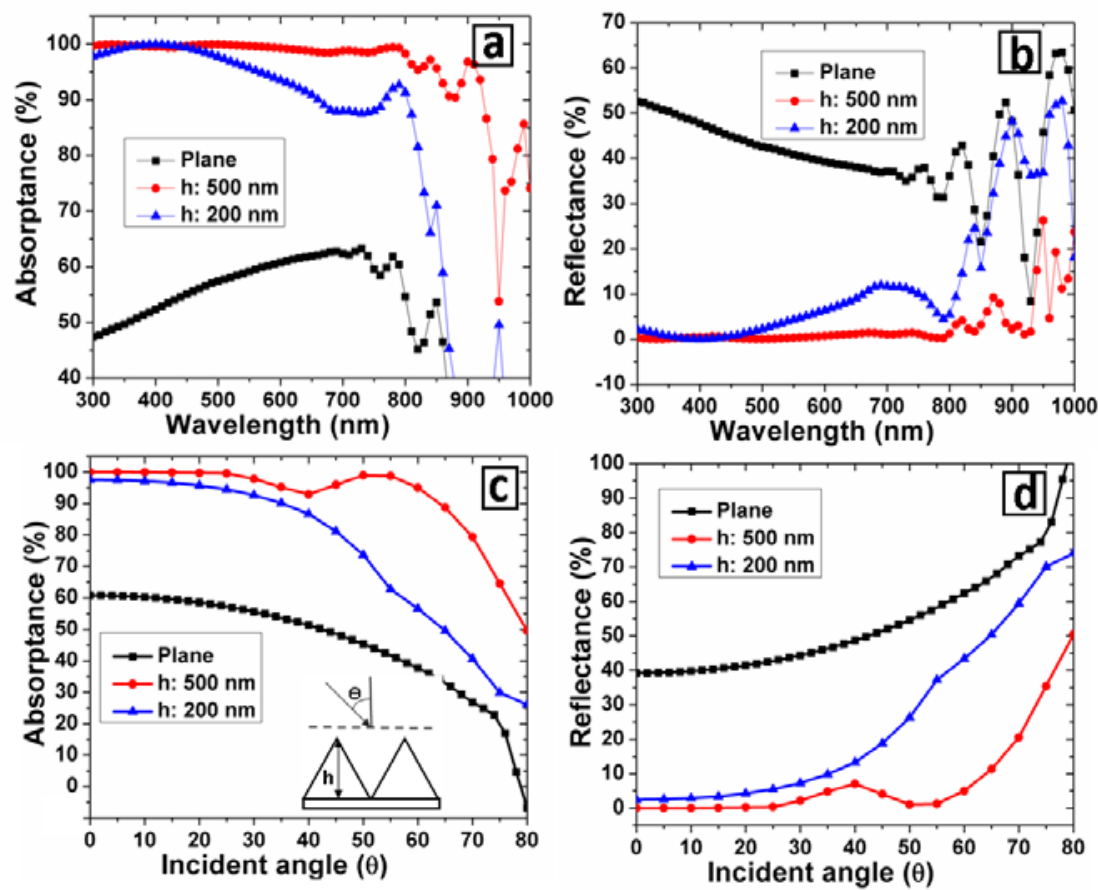

Fig. 7 Spectral (a) absorptance (b) reflectance with normal incidence of light and (c) absorptance (d) reflectance as a function of light incident angle for the wave length $400 \mathrm{~nm}$. The results are shown for flat cell, and two textured cells with different texture size. The inset figure in (c) shows texture dimensions used in these analysis.

due to the high dense textures and large crystal grains found in water treated samples [11].

Additionally, the experimental result is compared with simulation as shown in Fig. 7. For the numerical simulation, finite element method is used to solve the Maxwell's equation to model the laser induced structure. From AFM anal-

(a)

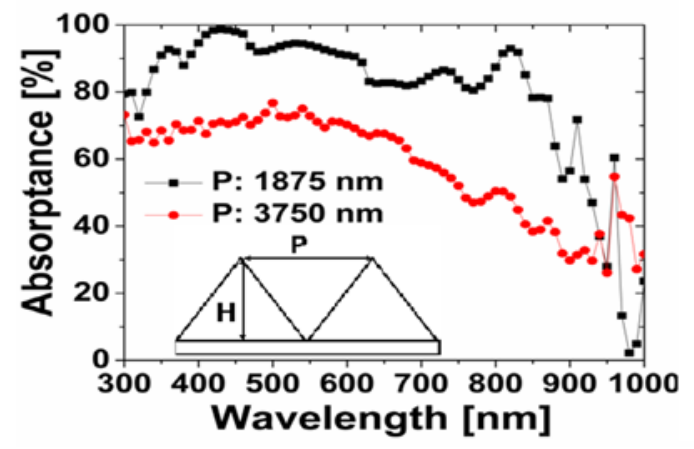

(b)

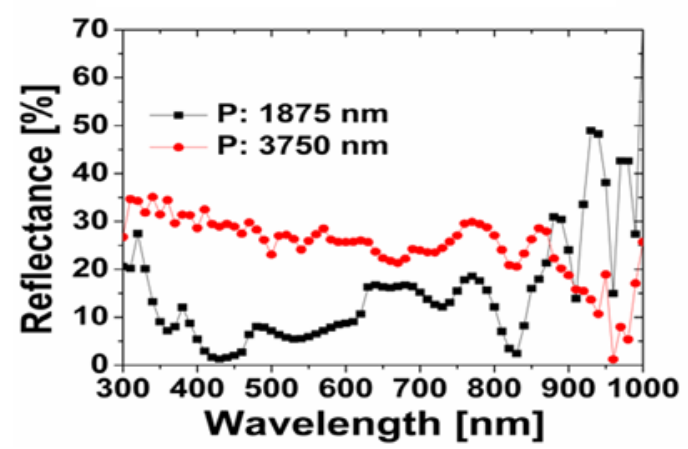

Fig. 8 Spectral (a) absorptance (b) reflectance as a function of different density for the texture height of $700 \mathrm{~nm}$ and base diameter of $1500 \mathrm{~nm}$. The inset figure in (a) shows texture dimensions used in these analvsis. ysis, the experimentally formed conical textures base dimeters are found to be $\sim 1500$ to $2500 \mathrm{~nm}$ for air and $\sim 1000$ to $2000 \mathrm{~nm}$ in water and the height values are in between $100 \mathrm{~nm}$ and $700 \mathrm{~nm}$ for both air and water. The average texture dimensions derived from AFM analysis are listed in Table 1. The texture height values $200 \mathrm{~nm}$ and $500 \mathrm{~nm}$ and base diameter $2000 \mathrm{~nm}$ which are in these range are chosen for the analysis. The conical textures formed with laser texturing are slightly randomized and has small order of multi scale structures, because of the slight variation of fluences and temperatures during the experimental process. In this study, since the exact random structure produced by laser texturing is difficult to reproduce or realize via simulation method, dimensions approaching the average size of the cone based on the experimental study are considered.

An unit cone bounded by periodical condition is irradiated with sunlight of wavelength ranging from $300 \mathrm{~nm}$ to $1100 \mathrm{~nm}$. The height and density of the cones are varied and angular and wavelength dependence of absorption and reflection are calculated. Figure 7(a) and (b) show the reflection and absorption spectra for the structure under analysis with normal incidence of light. The $1000 \mathrm{~nm}$ thick untextured plane surface serves as the reference. It is clear that light reflection decreases after incorporating the surface texturing, and the antireflection becomes more effective with increasing the height and absorption in the longer wavelength also increases. When the texture height increases from 200 to $500 \mathrm{~nm}$, the top angle of the conical texture decreases, increasing the number of multiple reflections, thus increasing the optical path length. Also, the longer wavelength of the incident light wave can easily penetrate through the small textures whereas taller textures produce reflections which enhances absorption. Silicon has week absorption at longer wavelength and this is the indi- 
cation that the increased optical path length with increased height allows for the silicon to absorb weekly absorbed photons with longer wavelength. Further analysis with increasing height has shown that after around 300 to $400 \mathrm{~nm}$ height, the integrated values of absorption and reflection over the silicon absorption spectrum of $300 \mathrm{~nm}$ to $1100 \mathrm{~nm}$ becomes almost constant, and the change in base diameter has little effect on these properties. These analysis clearly shows that by creating a texture of height $600 \mathrm{~nm}$ with laser texturing, we can achieve optimum efficiency. Figures 7(c) and (d) show lower incidence angles starting from normal incidence of light on the solar cell plane gives higher absorption and minimizes the reflection compared to other higher angular incidence.

The influence of texture density on absorptance and reflectance characteristics are studied by varying the periodicity of the textures. Figures 8(a) and (b) show spectral absorptance and reflectance as a function of different density for the texture height of $700 \mathrm{~nm}$ and base diameter of $1500 \mathrm{~nm}$. The density is changed by varying the peak to peak distance from $1875 \mathrm{~nm}$ to $3750 \mathrm{~nm}$. From the Figs. 8(a) and (b) it is clearly seen that absorption enhancement and reduction in reflection is more in high density textures compared to lower density textures, due to the increased scattering in high density structures. Recent studies on the random Si nanopyramids fabricated by etching process showed an improvement in light trapping efficiency in ultrathin c-Si (few tens of micrometers) [16]. Further analysis is essential to simulate light trapping and influence of wavelength in random structures.

\subsection{Open circuit voltage decay measurements}

The defects and dangling bonds in a-Si/poly-Si materials can cause a decrease in the carrier lifetime, which may in turn reduce the carrier diffusion length. This leads to reduction in the overall energy conversion efficiency. Carrier lifetime can be improved by annealing the a-Si and

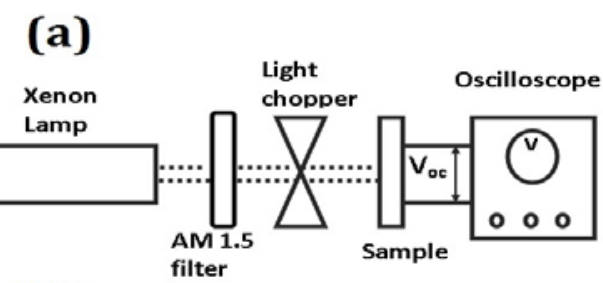

(b)

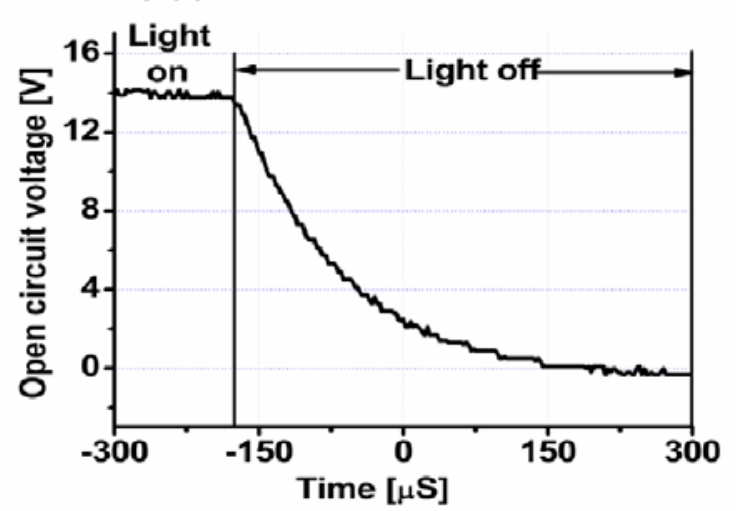

Fig. 9 (a) Experimental setup for open circuit voltage decay measurements (b) Open circuit voltage decay curve. when it is performed in water, due to very high laser induced temperature water molecules break in to hydrogen, oxygen and hydroxyl radicals [17]. This atomic hydrogen penetrates the film during annealing and attaches itself to defects and deactivate them. The open circuit voltage decay method is used for measuring the minority carrier lifetime [18]. The open-circuit voltage is measured as a function of time at the end of the light pulse, and by analysing the decay curve, the minority carrier lifetime can be calculated as shown in Fig. 9. AM 1.5 filtered Xe-lamp provided the light, cut down by light chopper (frequency $4000 \mathrm{~Hz}$ ) and the voltage-decay signal is captured using a $250 \mathrm{MHz}$ Digital storage Oscilloscope (Lecroy, wave runner). The experimental set-up is shown in Fig. 9(a).

The decay curve contains three regions of interest, high, intermediate and low levels of injection as reported in [14]. In the first two regions, the decay curve is linear whereas in the low level of injection, it becomes exponential as shown in figure 10 (a). The life time of minority carriers can be calculated with the slope of each region using the following equations:

$$
\begin{aligned}
& \frac{2 K T}{q}\left[\frac{d t}{d V o c}\right]-\text { Region } 1 \\
& \frac{K T}{q}\left[\frac{d t}{d V o c}\right] \quad-\text { Region } 2 \\
& \frac{K T}{q}\left[\exp \left[\frac{q V(0)}{K T}-1\right] \exp \left(\frac{-t}{\tau}\right)-\text { Region } 3\right.
\end{aligned}
$$

where the lifetime of the minority carrier is $\tau, K$ is Boltzmann's constant, $T$ is the absolute temperature, $q$ is the electron charge, $t$ is the time and $\frac{d V o c}{d t}$ is the slope of in each region and $V(0)$ is the open circuit voltage at the termination of excitation. Here we used intermediate region for the calculation of minority carrier life time and from which diffusion length is calculated as follows.

$$
\begin{array}{ll}
\text { Diffusion length: } & L=\sqrt{D \tau} \\
\text { Diffusivity : } & D=\mu \frac{K T}{q}
\end{array}
$$

where $\mu$ is the mobility of the minority carriers and the calculated minority carrier life times are shown in table 1. Water annealed sample shows 13\% improvement in the lifetime of minority carriers compared to air treated samples which indicates defects passivation with hydrogen in water.

Minority carrier diffusion length is important since it determine whether the carrier can cross the junction before recombination. Since our samples are n-type a-Si, minority hole mobility is considered. As per literature, it can vary from $0.01 \mathrm{~cm}^{2} / \mathrm{V}$-S to several hundred $\mathrm{cm}^{2} / \mathrm{V}$-S depending

Table 2 Minority carrier lifetime with the laser fluence $300 \mathrm{~mJ} / \mathrm{cm}^{2}$ with different spot overlap

\begin{tabular}{ccc}
\hline Ambience & 30 & 90 \\
\hline air & $30 \mu \mathrm{s}$ & $22.3 \mu \mathrm{s}$ \\
water & $33.45 \mu \mathrm{s}$ & $25.1 \mu \mathrm{s}$ \\
\hline
\end{tabular}




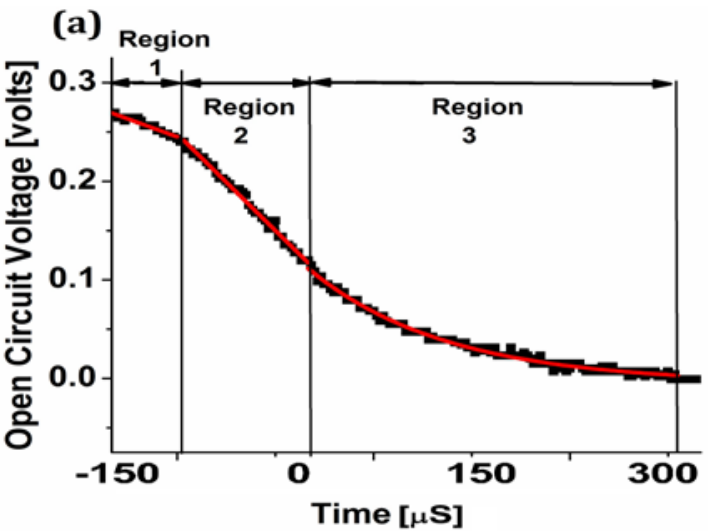

(b)

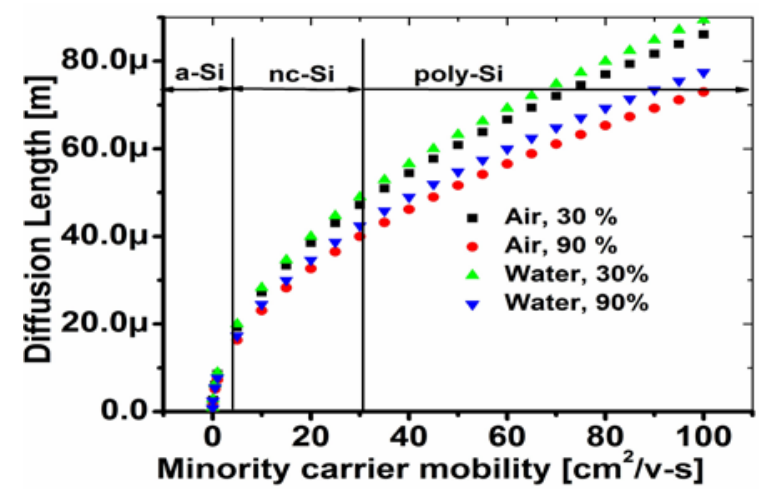

Fig. 10 (a) Open circuit voltage decay curve with three regions of interest (d) Diffusion length of minority carriers of the laser treated sample in air and water for $532 \mathrm{~nm}$ with $300 \mathrm{~mJ} / \mathrm{cm}^{2}$.

on the concentration. By considering these values, diffusion lengths for the calculated minority carriers are presented in Fig. 9(b). Even for the least possible value of 0.01 $\mathrm{cm}^{2} / \mathrm{V}$-S, which is in a-Si range, the diffusion length is around $900 \mathrm{~nm}$ and for poly-Si it is above $30 \mu \mathrm{m}$ which is sufficient for the carriers to cross the junction which is $1 \mu \mathrm{m}$ away from surface for our experimental samples.

\subsection{I-V characteristics}

Light I-V characteristics measurements are done by illuminating the sample using a Xe light source measuring the current and voltage with the precision source/ Measurement unit (B2901A, Keysight). The input intensity of the light at the sample surface is set to $60 \mathrm{~mW} / \mathrm{cm}^{2}$. The efficiency of the untreated a-Si sample is found to be around $0.013 \%$. After laser treatment, efficiency improves depending on the experimental conditions. Figures 11(a) and (b) show current - voltage measurements of laser treated samples in air and water for $50 \%$ of spot overlapping and the corresponding calculated values of efficiency for different percentage of spot overlap. Even though 90\% overlapped samples gives better light trapping textures with high roughness values, efficiencies of $30 \%$ and $50 \%$ are found to be as compared to that of $90 \%$ overlapping. This might be due to the removal of material and higher order of defects and damage inflicted on the sample due repeated irradiation of laser pulse on the same spot when treated with higher percentage of overlapping. The ablation threshold might have decreased with increasing number of pulses due to change in the nature and absorption behavior of the film after each laser pulse irradiation. Overall, there is a significant improvement in the absorption of incident light due to textured surface. Underwater treated samples gives better $\mathrm{I}-\mathrm{V}$ values compared to that of air treatment. These improved electrical characteristics in underwater annealing is an indication of high density light trapping structures and better crystallization.

\section{Conclusions}

Laser annealing and texturing with a Q-switched, nanosecond pulsed $\mathrm{Nd}^{3+}:$ YAG laser is performed and the formation of nano scale surface roughness in a-Si thin films that strongly enhances light-trapping with simultaneous crystallization is studied. Changes in surface morphology due to laser assisted texturing in air and water environments is observed using Optical profilometer, Scanning electron microscope and Atomic Force Microscopy. The light-trapping properties of textures are analyzed by optical reflectance measurements and compared with theoretical analysis. Theoretical analysis is performed by solving maxwell's equation and the incident angle and wavelength dependance of reflectance and absorptance is studied. Reduction in reflection is observed compared to planar surface indicating dependence of reflectance on surface textures which is in agreement with the theoretical analysis. The extent of crystallinity and the influence of structural characteristics on electronic properties are studied using I$\mathrm{V}$ characterization and the open circuit voltage decay method. After underwater annealing, the efficiency of naSi/p-cSi solar cells is found to be increased ( 2 to $3 \%)$
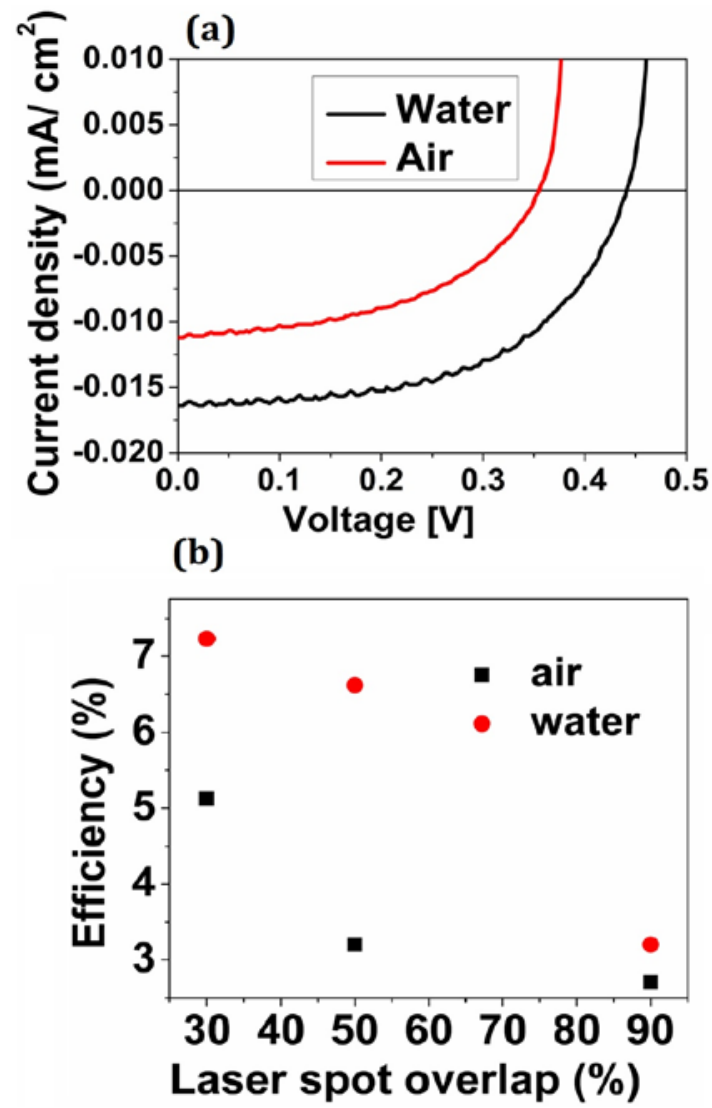

Fig. 11 (a) Current-voltage curve under illumination of the laser treated sample in air and water with $50 \%$ spot overlap and (b) efficiency for different percentage of spot overlap for $532 \mathrm{~nm}$ and fluence $300 \mathrm{~mJ} / \mathrm{cm}^{2}$. 
when compared to that in air and diffusion lengths of minority carriers above $1-\mu \mathrm{m}$ after laser treatment indicates the reduction in the probability of recombination before crossing the junction.

\section{Acknowledgments}

This work was supported by Department of Science and Technology, Government of India (Project \# SR/S3/MERC/0085/2010(G)). The authors are grateful to the members of Microelectronics and MEMS Laboratory, Department of Electrical Engineering, Scanning Electron Microscope and X-ray diffraction analysis Facilities in the Department of Metallurgy and Raman spectroscopy, Department of Physics of IIT Madras for providing the facilities for characterization.

\section{References}

[1] X. Deng and E.A. Schiff : "Amorphous Silicon Based Solar Cells" ed. by A. Luque and S. Hegedus, (John Wiley \& Sons, Chichester, 2003) p.505.

[2] G. N. Tiwari and R. K. Mishra: "Advanced Renewable Energy Sources" (Royal Society of Chemistry, London, 2011) p.60

[3] N.H .Nickel: "Laser crystallization of silicon" (Elsevier, Academic Press, Amsterdam,2003) p.3.

[4] Y. E. B. Vidhya, R.Sriram and N. J. Vasa J. Laser Micro/Nanoengin. 10, (2015) 334.

[5] H.Azuma, A.Takeuchi, T,Ito, H.Fukushima, T.Motohiro and T.Yamaguchi: Solar Energ. Mater. Solar Cells, 74, (2002) 289.
[6] K.C. Chyuan, Y.W. Chang, L.J. Feng and J.J. Ywan: Thin Solid Films, 515, (2007) 8094.

[7] I.A. Palani, N.J. Vasa, M. Singaperumal, T. Okada,T: Thin Solid Films, 518, (2010) 4183.

[8] C.H. Crouch, J.E. Carey, J.M. Warrender, M.J. Aziz, E. Mazur and F.Y. Genin: Appl. Phys. Lett., 84, (2004) 1850.

[9] V.V. Iyengar, B.K. Nayak, K.L. More, H.M. Meyer, M.D. Biegalski, J.V. Li and M.C. Gupta: Solar Energ. Mater. Solar Cells, 95, (2011) 2745.

[10]B.K. Nayak and M.C. Gupta: Appl. Phys. A, 89, (2007) 663.

[11]Y. E. B. Vidhya and N. J. Vasa: J. Photon. Energy 6 , (2016) 014001.

[12]Y. E. B. Vidhya and N. J. Vasa: Procedia Manufacturing, 5, (2016) 734.

[13] J. Li, H. Y. Yu, Y. Li, F. Wang, M. Yang and S. M. Wong: Appl. Phys. Lett., 98, (2011) 021905.

[14] S. I. Dolgaev, S. V. Lavrishev, A. A. Lyalin, A. V. Simakin, V. V. Voronov and G. A. Shafeev: Appl. Phys. A: Mater. Sci. Process., 73, (2001) 177.

[15] M. Y. Shen, C. H. Crouch, J. E. Carey, R. Younkin, E. Mazur, M. Sheehy and C. M. Friend: Appl. Phys. Lett. 82, (2003) 1715.

[16]S. Zhong, W. Wang, Y. Zhuang, Z. Huang and W.Z. Shen: Adv. Funct. Mater., 26, (2016) 4768.

[17]Y. E. B. Vidhya and N. J. Vasa: Appl. Phys. A 123, (2017) 528.

[18] J. E. Mahan, T. W. Ekstedt, R. I. Frank and R. Kaplow: IEEE Trans. Electron Dev., 26, (1979) 733. 